\title{
A Survey on Enhancing Routing using NCPR in Mobile Ad hoc Networks
}

\author{
Smita S Shrimandal; Ranjana B Nadagoudar; Rekha B Venkatapur \\ M.Tech $4^{\text {th }}$ SEM, Department of CS, VTU PG Center Regional Office, Gulbarga \\ Assistant Professor, Department of CSE, VTU PG Center Regional Office, Gulbarga \\ Professor and Head, Department of CS, K S Institute of Technology, Bangalore
}

\begin{abstract}
Mobile Ad hoc Network (MANET) is collection of wireless mobile hosts (or nodes) that are free to move in any directions at any speed. This nature of MANET's leads to periodic link breakage, which in turn leads to periodic path failure and route discoveries, hence overhead is created in the network topology. To overcome this overhead basic technique called Broadcasting is used which is inefficient in larger topologies as it incurs broadcast storm problems. This paper surveys and presents implementation of proposed NCPR Protocol to enhance routing performance by reducing the overhead. The NCPR Protocol determines ReBroadcast order using ReBroadcast delay by using neighbour coverage knowledge. Approach is to overcome collisions and channel contentions by reducing the number of retransmission and improve the Quality of Service $(Q o S)$ routing in MANETS.
\end{abstract}

General Terms: Broadcast, Routing, collision, Mobility, channel contentions.

Keywords: Mobile Ad hoc Networks (MANETs), Neighbour coverage Knowledge, ReBroadcast

Techniques, Routing Overhead, NCPR.

\section{Introduction}

Mobile Ad hoc Network (MANET) is collection of wireless mobile hosts (or nodes) that are free to in any directions at any speed. Mobile nodes are equipped with a wireless transmitter and a receiver that communicate directly with each other or forward message through other nodes.

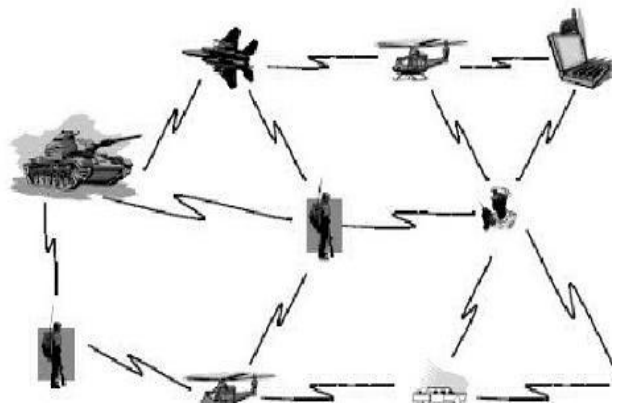

Fig. 1 Mobile Ad hoc Network

One of the major advantages of mobile networks is to allow different nodes for data communications and still maintain their mobility. However, this communication is limited to the range of transmitters. It means that two nodes cannot communicate with each other when the distance between the two nodes is beyond the communication range of their own. Broadcasting is a common problem in networks. In ad-hoc networks, broadcast plays an important role, relaying message generated by one node to all other nodes. Broadcasting[1] is an integral part of a variety of protocols that provide basic functionality and efficiency to higher-layer services. MANETs consists of collection of nodes which can move freely. No base stations are supported in MANETs. These nodes can be dynamically self-organized into arbitrary topology networks without a fixed infrastructure. One of the fundamental challenges in the MANETs is the design of dynamic routing protocols with good performance and less overhead [2]. Routing protocols in ad-hoc networks are classified into proactive (tabledriven) protocol and reactive (on demand) protocol

1. Proactive protocols maintain routes between every host pair at all times. In proactive protocol, when a packet needs to be forwarded the route is already known. This protocol is based on periodic updates. They maintain up-to-date routing information for all nodes in the network even before it is needed. Because of this situation, proactive protocols will require low latency. This protocol incurs more overhead. Examples of this type include OLSR and DSDV routing protocol.

2. Reactive routing protocols do not maintain routing information at the nodes if there is no activity between 
them. (i.e.) it determines the route if and when needed. This protocol incurs less overhead. Examples of this type include AODV[3], DSR routing protocol. Nodes in the reactive routing protocols are trying to minimize the overhead by only sending routing information as soon as the communication is initiated between them Some methods have been proposed to optimize the broadcast problem in MANETs in the past few years. Williams and Camp [4] categorized broadcasting protocols into four classes:

1. Simple flooding

2. Probability based methods

3. Area based methods

4. Neighbour knowledge methods

For the above four classes of broadcasting protocols, they showed that an increase in the number of nodes in a static network will degrade the performance of the probability based and area based methods [5]. Our approach combines the advantages of the neighbour coverage knowledge and the probabilistic mechanism, which can decrease the number of retransmissions so as to reduce the routing overhead and enhance the routing performance.

\subsection{AODV: Ad hoc On-demand Distance Vector Routing}

\section{Review of Routing Protocols in MANET's}

On-demand routing protocols [3] construct a path to a given destination only when it is required. They do not maintain topological information about the whole network, and thus there is no periodic exchange of routing information. They broadcast a Route REQuest (RREQ) packet to the networks, and the broadcasting induces excessive redundant retransmissions of RREQ packet and causes the broadcast storm problem [6], which leads to a considerable number of packet collisions, especially in dense networks [7]. Therefore, it is indispensable to optimize this broadcasting mechanism.

\subsection{NCPR}

The propose method NCPR is to determine ReBroadcast delay in the ReBroadcast order, and obtain the more exact additional coverage ratio by sensing neighbour coverage knowledge. In this section, the ReBroadcast delay and ReBroadcast probability of the proposed protocol is calculated.

\section{1) ReBroadcast Delay}

The ReBroadcast delay is to find out the forwarding order. The node has lower delay if it has more common neighbours with the previous node. If this node ReBroadcast s a packet by which further common neighbours will know this information. Node pi has more neighbours uncovered by the RREQ packet from $s$. The meaning of that if node pi ReBroadcast s the RREQ packet, then it can reach more neighbour nodes. To quantify of the Uncovered Neighbours (UCN) set U(pi) of node pi as follows:

$$
\mathrm{U}(\text { pi })=\mathrm{N}(\text { pi })-[\mathrm{N}(\text { pi }) \cap \mathrm{N}(\mathrm{s})]-\{\mathrm{s}\}
$$
(1)

The N(s) and N(pi) are the neighbours sets of node. $\mathrm{s}$ is sends an RREQ packet to node pi. According to Eq.

In order to overcome the channel collision every node must be find the ReBroadcast delay. When we find the ReBroadcast delay the protocol affect the neighbour conformation knowledge. So any node will calculate the delay by RREQ packet. The node calculates by checking neighbour list of RREQ packet and itself neighbour list. The ReBroadcast delay Td(pi) of node pi is defined as follows:

$$
\begin{aligned}
& \mathrm{Tp}(\text { ni })=1-\mid \mathrm{N}(\mathrm{s}) \cap \mathrm{N}(\text { ni }) \mid(1) \\
& |\mathrm{N}(\mathrm{s})| \mathrm{T} \text { d (pi) = Max Delay } \times \mathrm{Tp}(\text { pi })
\end{aligned}
$$

Where Tp (pi) is the delay ratio of node pi, and MaxDelay is a small constant delay. $|\cdot|$ is the number of elements in a set. The objective of this ReBroadcast delay is not to ReBroadcast the RREQ packet to more nodes, but to disseminate the neighbour coverage knowledge more quickly. After determining the ReBroadcast delay, the node can set its own timer.

\section{2) ReBroadcast Probability}

The ReBroadcast probability is collection of two factors:

a) Additional coverage ratio: It is the ratio of the number of nodes that should be covered by a single broadcast to the total number of neighbours 
b) Connectivity factor: It is the relationship of network connectivity and the number of neighbours of a given node that are additionally covered by the node which has a more ReBroadcast delay might listen to RREQ packets from the nodes.

The additional coverage ratio $\mathrm{CR}$ (ni):

$$
\mathrm{CR}(\mathrm{ni})=|\mathrm{U}(\mathrm{ni})||\mathrm{N}(\mathrm{ni})|
$$

Connectivity factor can be defined as:

$$
\mathrm{CF}(\text { ni) }=\mathrm{Nc}|\mathrm{N}(\mathrm{ni})|
$$

$\mathrm{Nc}=5.1774 \log \mathrm{n}$, the $\mathrm{n}$ is the number of nodes [8] in the network.

Combining the additional coverage ratio and connectivity factor, to obtain the ReBroadcast probability RBP (ni) of node ni:

$\mathrm{RBP}(\mathrm{ni})=\mathrm{CF}(\mathrm{ni}) \cdot \mathrm{CR}(\mathrm{ni})$

Where, if the RBP (ni) is > 1, to set the RBP (ni) to 1 .

Below fig 2 defines the NCPR algorithm.

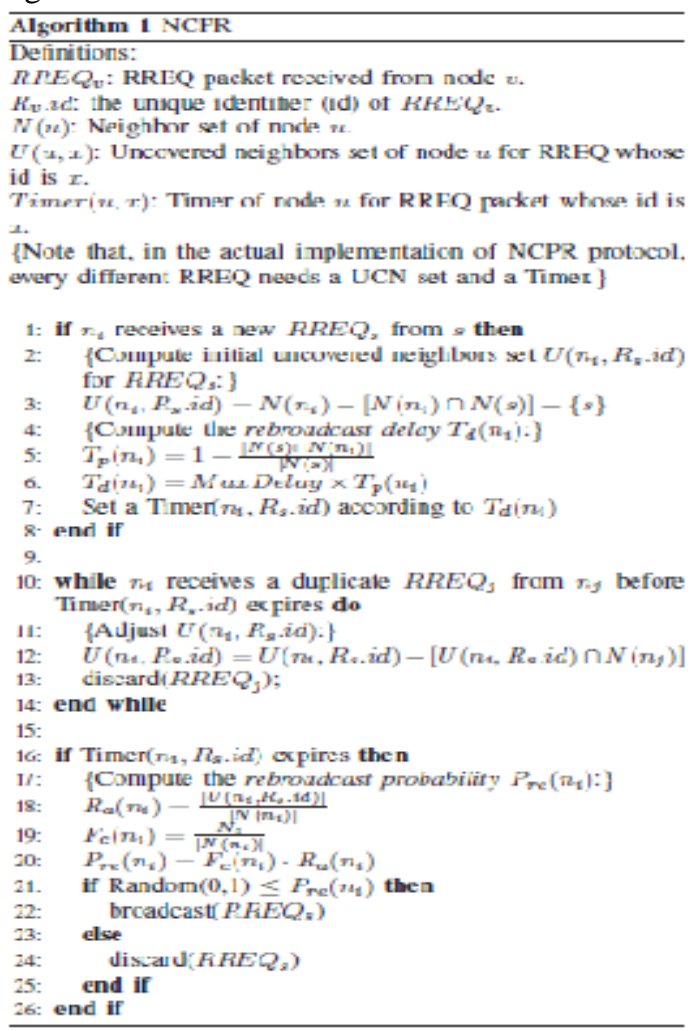

Fig. 2 Algorithm used for NCPR

\section{System Architecture for NCPR}

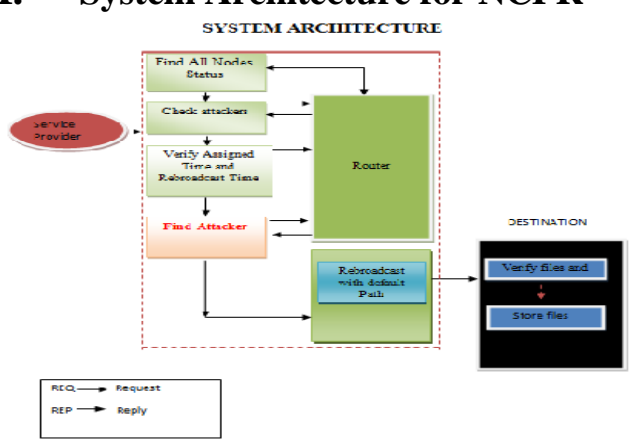

Fig. 3 System Architecture for NCPR 
In the network topology, the nodes from source to destination are scattered and the route discovery is made using ReBroadcast Delay along with ReBroadcast Probability Fig 3 describes the System architecture used for NCPR where in the status of the nodes are checked and routing time are assigned and data are uploaded which needs to be sent to destination, router is the main unit here which computes ReBroadcast delay and maintains backlog on attacks and finally sends data to destination. If the routing time exceeds the assigned time the ReBroadcast has to take place which here is default path

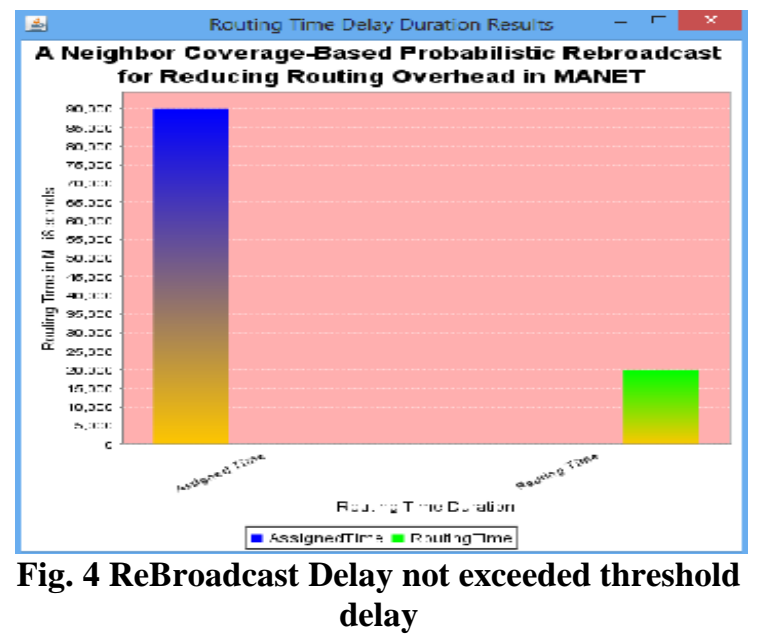

To calculate ReBroadcast delay, the choice of proper delay is must and it is key to success for the NCPR as the delay time affects dissemination of neighbour coverage knowledge, hence for given time the ReBroadcast delay is calculated for the network topology. If the computed ReBroadcast delay is less than the given time it indicates that routing time taken by NCPR is less than assigned time as depicted in Fig 4

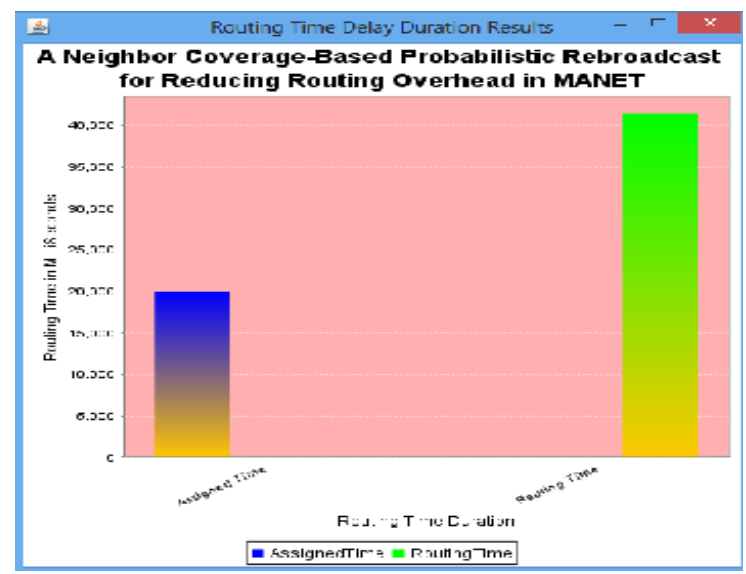

Fig. 5 ReBroadcast Delay exceeds threshold delay

Above Fig 5 describes the ReBroadcast delay calculated for the topology is more than the given time hence the routing time is huge compared to assigned time and this leads to link breakage which results in ReBroadcast

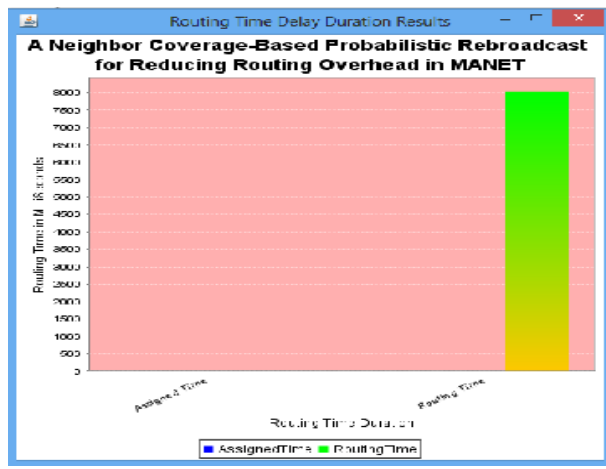

Fig. 5 Attack 
The above Fig 5 depicts for given no assigned time, which leads to more routing time and prone to vulnerable attacks

\section{Conclusion and Future Directions}

In this survey paper, The NCPR protocol a new scheme to dynamically calculate the ReBroadcast delay, which is used for calculation of the forwarding order and more effectively use the neighbour coverage knowledge. Because of less redundant rebroadcast, the proposed protocol mitigates the network collision and generates less rebroadcast traffic than the flooding and some other optimized scheme in literatures. For further improvements in implementations and performance within dense networks as future work.

\section{References}

[1] S. Y. Ni, Y. C. Tseng, Y. S. Chen, and J. P. Sheu. "The Broadcast Storm Problem in a Mobile Ad hoc Network," Proc. of ACM/IEEE MobiCom'99, pp. 151-162, 1999.

[2] Xin Ming Zhang, En Bo Wang, Jing Xia, and Dan Keun Sung."A Neighbour Coverage based Probabilistic ReBroadcast for Reducing Routing Overhead in Mobile Ad hoc Networks", 2012.

[3] C. Perkins, E. Belding-Royer, and S. Das, "Ad hoc On-Demand Distance Vector (AODV) Routing," RFC $3561,2003$.

[4] B. Williams and T. Camp, "Comparison of Broadcasting Techniques for Mobile Ad Hoc Networks," Proc. ACM MobiHoc'02, pp. 194-205, 2002.

[5] J. Kim, Q, Zhang, and D. P. Agrawal, "Probabilistic Broadcasting Based on Coverage Area and Neighbour Confirmation in Mobile Ad hoc Networks," Proc. of IEEE GLOBECOM'04, 2004.

[6] Y. C. Tseng, J. P. Sheu Y. S. Chen, and S. Y. Ni,. “The broadcast storm problem in MANET,” 1999, ACM/IEEE pp. 151-162.

[7] A. Mohammed, M. Ould-Khaoua, L.M. Mackenzie, C. Perkins, and J D. Abdulai, "Probabilistic Counter-Based Route Discovery for Mobile Ad Hoc Networks," Proc. of IWCMC'09, pp. 1335-1339, 2009.

[8] F. Xue and P.R. Kumar, "The Number of Neighbours Needed for Connectivity of Wireless Networks,” Wireless Networks, vol. 10, no. 2, pp. 169-181, 2004. 